\title{
Comparisons of psychophysical and neurophysiological studies of cochlear implants
}

\author{
Bryan E. Pfingst \\ Kresge Hearing Research Institute, Department of Otorhinolaryngology, University of Michigan, Ann Arbor, Michigan, U.S.A.
}

(Received 30 October 1987: accepted 7 March 1988)

\begin{abstract}
This paper compares psychophysical and neural studies of electrical stimulation of the auditory nerve with the goal of evaluating the relevance of single-unit animal models for the development of cochlear prostheses for profoundly deaf humans. Comparative psychophysical studies with implanted deaf subjects indicate that animal models, at least nonhuman primates, provide a close match to humans, though this is not always true for acoustic stimulation of normal-hearing subjects. However, the human-animal comparisons, especially those involving electrical stimuli, need further study using more carefully matched conditions. Comparisons of psychophysical and neurophysiological thresholds for electrical stimulation in animals reveal consistently higher thresholds in the neural studies. A number of factors which may account for these differences are discussed. A partial resolution of the problem could result from conducting neurophysiological and behavioral studies in the same animal. Finally, comparison of psychophysical and neurophysiological studies of temporal encoding suggest that there may be more information encoded in the auditory nerve than is used by the system, at least for nonspectral frequency discrimination. This points to a need for further analysis of the processing of this information at higher levels in the auditory pathway.
\end{abstract}

Biological models; Nonhuman primates; Cochlear prosthesis; Psychophysics; Neurophysiology; Single units

\section{Introduction}

One of the newest applications of the single-unit animal model, and one of the most exciting in terms of its potential benefit to clinical otolaryngology, involves the neurophysiological study of cochlear implants. At least five laboratories around the world are actively engaged in single-unit studies involving electrical stimulation of the auditory nerve. It seems appropriate therefore to evaluate this animal model using some of the approaches discussed in this workshop. I will begin with the comparative psychophysics approach.

\section{Comparative psychophysics}

For acoustic stimuli, the nonhuman mammals typically used for single-unit recording are roughly equal to or better than humans in detecting stimuli,

Correspondence to: Bryan E. Pfingst, Kresge Hearing Research Institute, Box 0506, University of Michigan Medical School, Ann Arbor, MI 48109, U.S.A. particularly at high frequencies, but are typically worse than humans in discriminating frequency or intensity changes (Fay, 1988). For electrical stimulation the picture is quite different. The evidence to date (detailed below) indicates little or no difference in performance between humans and animals on any of these tasks. Note that conclusions at this time must be limited to human versus nonhuman primates, as data on the psychophysics of electrical stimulation for other nonhuman mammals is sparse. Given the neuroanatomic data on species differences in the auditory nerve and CNS auditory pathways (Nadol, 1988; Moore, 1987), it should not be assumed that the monkey data will apply to other mammals. Additional psychophysical studies are needed in other species, particularly cats and guinea pigs which are commonly used for single-unit studies.

Comparison of psychophysics across animals which differ anatomically may lead to insights as to the functional role of the anatomical structures. Particularly intriguing is the presence of reciprocal synapses on outer hair cells which seem to occur normally only in higher primates (Nadol, 1988). 
As Fay (1988) pointed out, comparison of hearing across species, which in many cases means comparison across laboratories, is fraught with difficulties. Nevertheless, there is sufficient consistency across a number of studies to be convincing, at least with regard to the acoustic signals discussed by Fay. With cochlear implants the problems are worse because the numbers are smaller, and because in many cases sufficient details of the experimental procedures do not appear in the literature. Nevertheless, the findings to date point to similarities between human and nonhuman performance more strongly than they point to differences. I will review those data briefly below.

Thresholds for electrical stimulation of human and nonhuman-primate subjects are compared in Fig. 1 and 2. The six nonhuman primate cases were chosen to represent a range of nerve survival patterns comparable to that seen in humans by
Hinojosa and Marion (1983) and represents the full range of thresholds we have seen in 30 cases where nonhuman primates with scala tympani implants in deaf ears were tested psychophysically. Thresholds for human subjects obtained by other investigators (Fig. 2) show similar shapes and cover a similar range of currents. One difference between these two sets of data is the higher slopes of some human threshold contours between about $60 \mathrm{~Hz}$ and $200 \mathrm{~Hz}$. This deserves further study.

Dynamic ranges for electrical stimuli also assume similar values in human and nonhuman primates. In both species, dynamic ranges are larger at low frequencies (around $100 \mathrm{~Hz}$ ) than at high frequencies (above $500 \mathrm{~Hz}$ ) (see review by Pfingst, 1984). Data at this time are insufficient to allow more detailed comparisons.

Level difference limens for electrical stimuli, obtained from monkeys, show a clear dependence on the frequency and level of stimulation (Pfingst

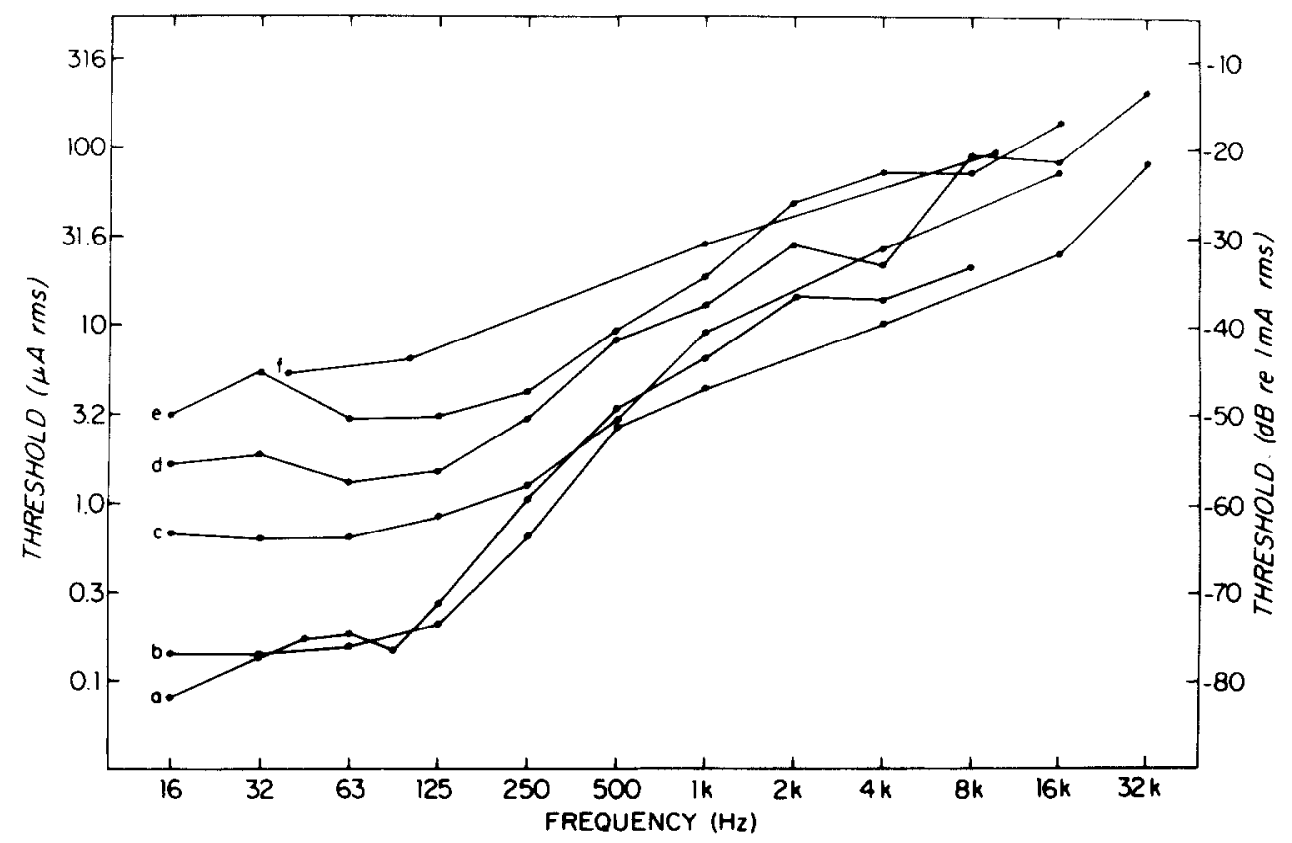

Fig. 1. Threshold contours for six monkeys with cochlear implants. Detection thresholds for sinusoidal electrical stimulation are plotted as a function of stimulus frequency. The monkeys were implanted with multielectrode scala tympani (hasal turn) implants and these thresholds are for bipolar stimulation between electrodes spaced 2.0 to $3.7 \mathrm{~mm}$ apart. Thresholds for monopolar stimulation (where tested) were similar. The cochleas were poisoned by local perfusion of neomycin sulfate through the round window just prior to implantation. These contours were measured after the thresholds became stable. The thresholds were rechecked just prior to sacrifice and found to be at the same levels shown here. These six cases were selected to represent a wide range of nerve survival patterns. Case $a$ had the best nerve survival (about $50 \%$ of the normal complement of spiral ganglion cells remaining in the basal region and $80-100 \%$ remaining in the apex) while case $f$ had the worst (less than $20 \%$ survival throughout the cochlea). (From Pfingst et al., 1985) 




Fig. 2. Threshold contours for six human cases. Thresholds for monopolar sinusoidal stimulation of electrodes in the basal turn of the scala tympani are shown. The subjects' initials, electrode designations, and sources of the data are shown in the inset.

et al., 1983) (Fig. 3). Earlier published reports on implanted humans stated that electrical level difference limens were constant across frequency and level, suggesting a significant species difference * However, more recent data by Shannon (1983) (Fig. 4) show the same relationships as those seen in the data from monkeys. Furthermore, the ranges of level difference limens for the two species largely overlap.

Frequency difference limens for electrical stimuli for humans and monkeys also overlap. Fig. 5 compares human data from the literature (open symbols) and monkey data from our laboratory (filled circles). A wide range of values for human subjects are found in the literature but the data for nonhuman primate fall in the middle of this range. These comparisons are complicated by several factors. There is ambiguity regarding the level at which some of the human data were collected. Clearly, stimulus level significantly af-

* It is possible that these earlier statements regarding electrical intensity difference limens were based on too little or too variable data. It is also possible that for selected stimulus parameters level difference limens are constant across level (White, 1984). This later possibility deserves further study.

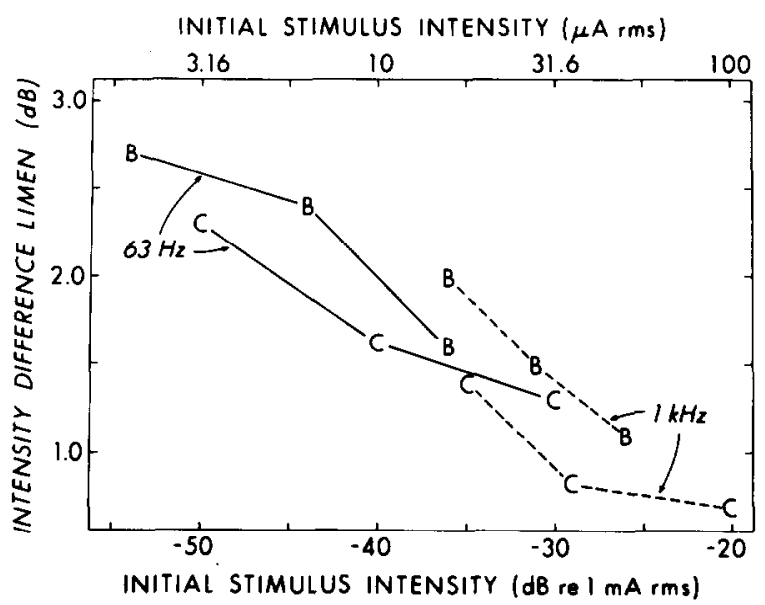

Fig. 3. Level difference limens for two monkeys (B and $C$ ) at two different frequencies of sinusoidal stimulation. Initial stimulus intensities are at $25 \%, 50 \%$ and $75 \%$ of the dynamic range $\pm 10 \%$. (From Pfingst and Sutton, 1983)

fects the magnitude of frequency difference limens, as illustrated in Fig. 5 by the data for the monkey at three levels at $100 \mathrm{~Hz}$. Furthermore, there is uncertainty about whether the human subjects were using loudness cues. In studies in monkeys we have found that deviations in level 


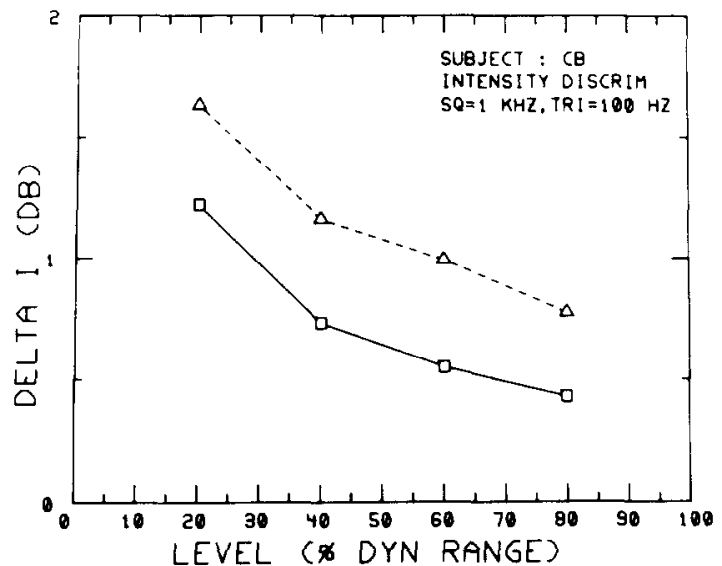

Fig. 4. Level difference limens for a human subject at two different frequencies of sinusoidal stimulation, plotted as a function of initial stimulus level. Dynamic range was $30 \mathrm{~dB}$ at

$100 \mathrm{~Hz}$ and $18 \mathrm{~dB}$ at $1 \mathrm{kHz}$. (From Shannon, 1983)

from the equal-loudness point by only a few tenths of $\mathrm{a} \mathrm{dB}$ are sufficient to significantly increase the percent hits in a frequency-level discrimination task (Pfingst and Rush, 1987). We suspect that in most frequency discrimination experiments with implanted human subjects loudness has not been matched to a degree sufficient to completely eliminate loudness cues, which therefore suggests that some of the human frequency difference limens reported in the literature are artificially low. This reinforces our view that monkeys do at least as well as humans in discriminating changes in the frequencies of electrical sinusoids.

Fig. 6 summarizes data from a number of studies on the discrimination of electrical and acoustic frequency changes by humans and monkeys. Ranges of values obtained across subjects by various laboratories are shown. For the acoustic data, perhaps the best comparison between the monkeys and humans for our purposes would use the data from Spiegel and Watson for non-musicians (range $c)$. These subjects received little training in the task and thus would be expected to perform no better, on the basis of training, than cochlcar implant subjects. Even under these less than ideal conditions humans perform better than welltrained monkeys in the majority of cases. The data for the monkeys (range $d$ ) overlap only the upper $20 \%$ of the range of the human data (on a log scale) and extend beyond this range. In contrast, when the performance of a subset of these same

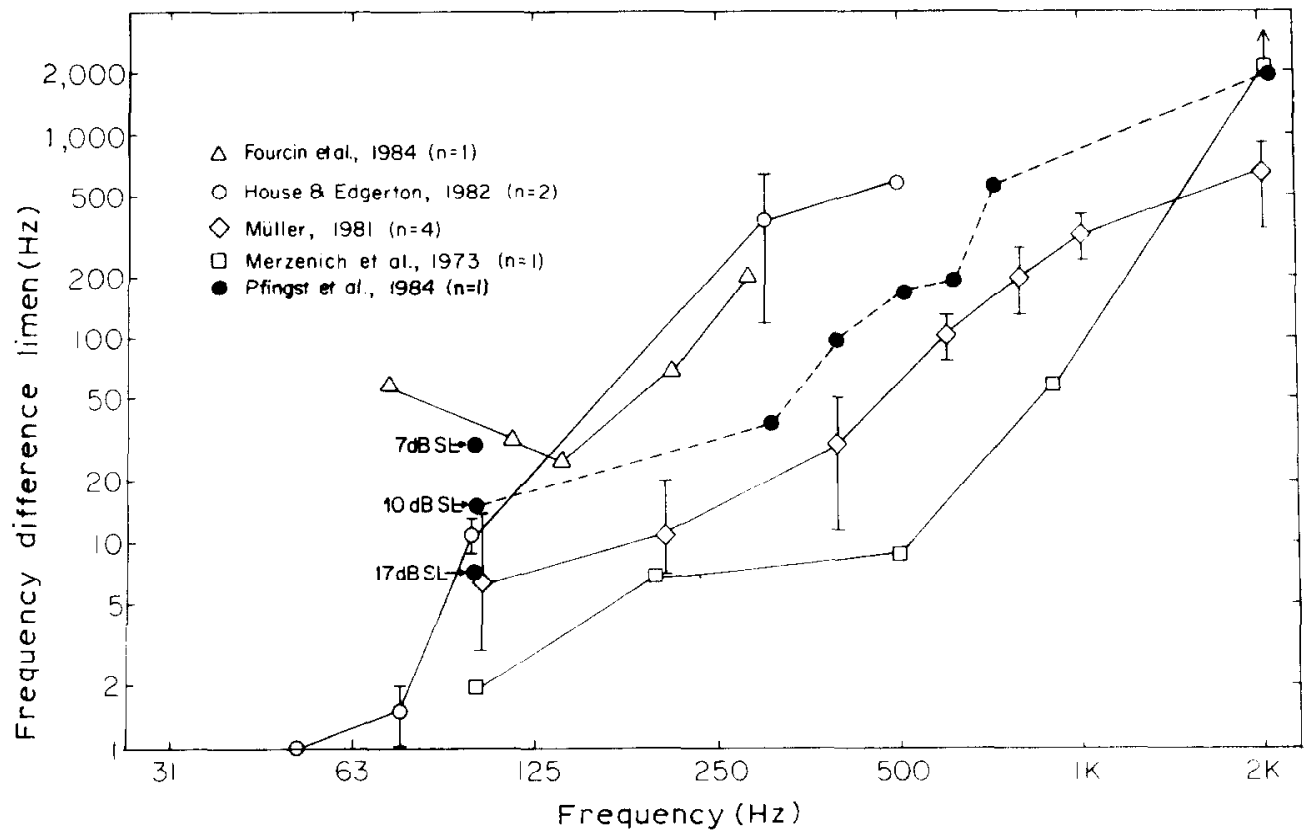

Fig. 5. Frequency difference limens as a function of initial stimulus frequency for human (open symbols) and nonhuman (filled symbols) primates. Sources of the data and number of subjects are shown in the inset. 


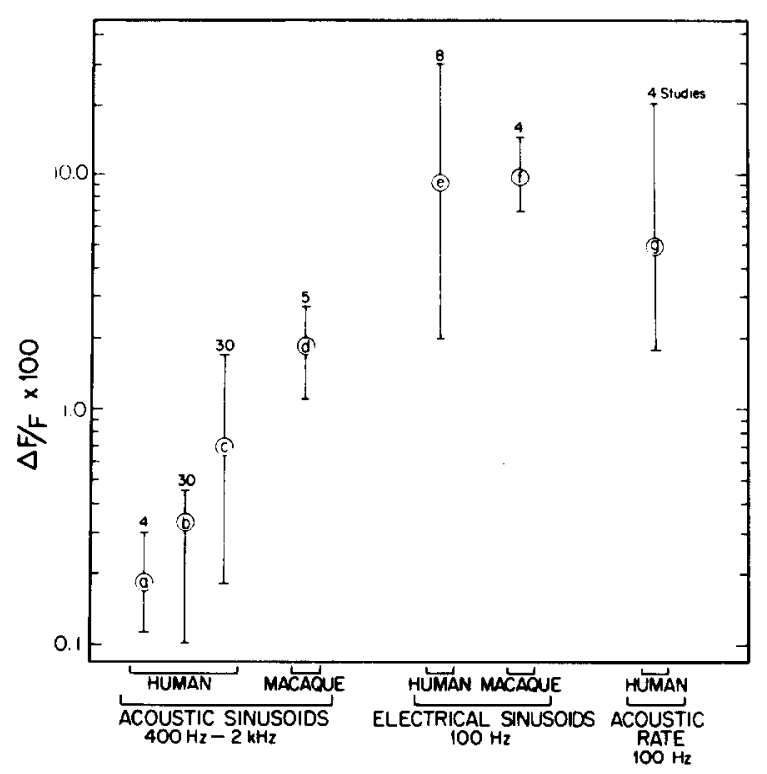

Fig. 6. Ranges of frequency difference limens obtained across subjects from studies in humans and monkeys as indicated below. The number of subjects from each study is indicated at the top of each range except in case $g$ which represents the range of means obtained across four studies: $a$-from Wier et al., 1977; $b$-musicians from Spiegel and Watson, 1984; $c$-nonmusicians from Spiegel and Watson, 1984; $d$-unpublished acoustic data from 5 monkeys-Pfingst; $e$-from human subjects in Fig. $5 ; f$-data for electrical sinusoids from 4 of the 5 monkeys whose acoustic data is shown in $d$, from Pfingst and Rush, 1987 plus unpublished data; $g$-data from 4 studies summarized by Formby, 1985, Fig. 2.

monkeys on an electrical frequency-discrimination task (range $f$ ) is compared with data from the literature on electrical frequency discrimination in humans (range $e$ ), the monkey data fall approximately in the middle of the range of values reported for the humans. Interestingly, this range is also close to that reported for humans using acoustic signals which lack spectral cues for the frequency change (range $g$ ).

Obviously, when comparisons of this sort are made using data from different laboratories collected under different conditions there is a risk that the results are due to differences in procedure rather than species. However, some of these comparisons have been made in the same laboratory using the same equipment and procedures. Under these conditions, Sinnott et al. (1985) found that monkeys had higher pure tone frequency difference limens than humans. Long and Clark
(1984) studied difference limens for the frequencies of pure tones and SAM noise in chinchillas and humans and concluded that the human-animal differences are much smaller for nonspectral frequency discrimination than for frequency-discrimination involving spectral cues. Clearly we need additional work in the area of electrical frequency discrimination. The studies should be conducted using human and monkey subjects which are closely matched with regard to stimulus parameters, implant type, thresholds, dynamic ranges, and degree of training. Procedures and equipment should be matched as closely as possible. However, if forced to make a choice at this point I would say that the evidence is in favor of little or no difference between human and monkey performance on these tasks for electrical stimulation.

These comparisons are rather important for interpreting the neurophysiological data from both normal-hearing animals and deafened, electrically stimulated animals. They suggest the interesting hypothesis that the monkey-human differences observed with acoustic stimulation may relate to differences in the spectral analysis or in the central processing of place information. They also suggest that the responses of these two species to temporal information may be similar. If this conclusion is correct then we are justified in using psychophysical data from either humans or monkeys to interpret the single-unit data obtained on temporal encoding of frequency information.

\section{Comparison of neurophysiology to psychophysics}

\section{Thresholds}

I turn now to a consideration of neurophysiological studies which have been done with cochlear implants, and the relation of these to the psychophysical data. Again, I will begin with a consideration of thresholds. Fig. 7 compares behavioral thresholds for six monkeys with varying degrees of nerve survival, as in Fig. 1, with ranges of thresholds of auditory nerve fibers or anteroventral cochlear nucleus units from published data from five different laboratories. The neural thresholds are plotted as a function of the stimulus frequency for sinusoidal stimulation or the inverse of the pulse period for pulsatile stimula- 


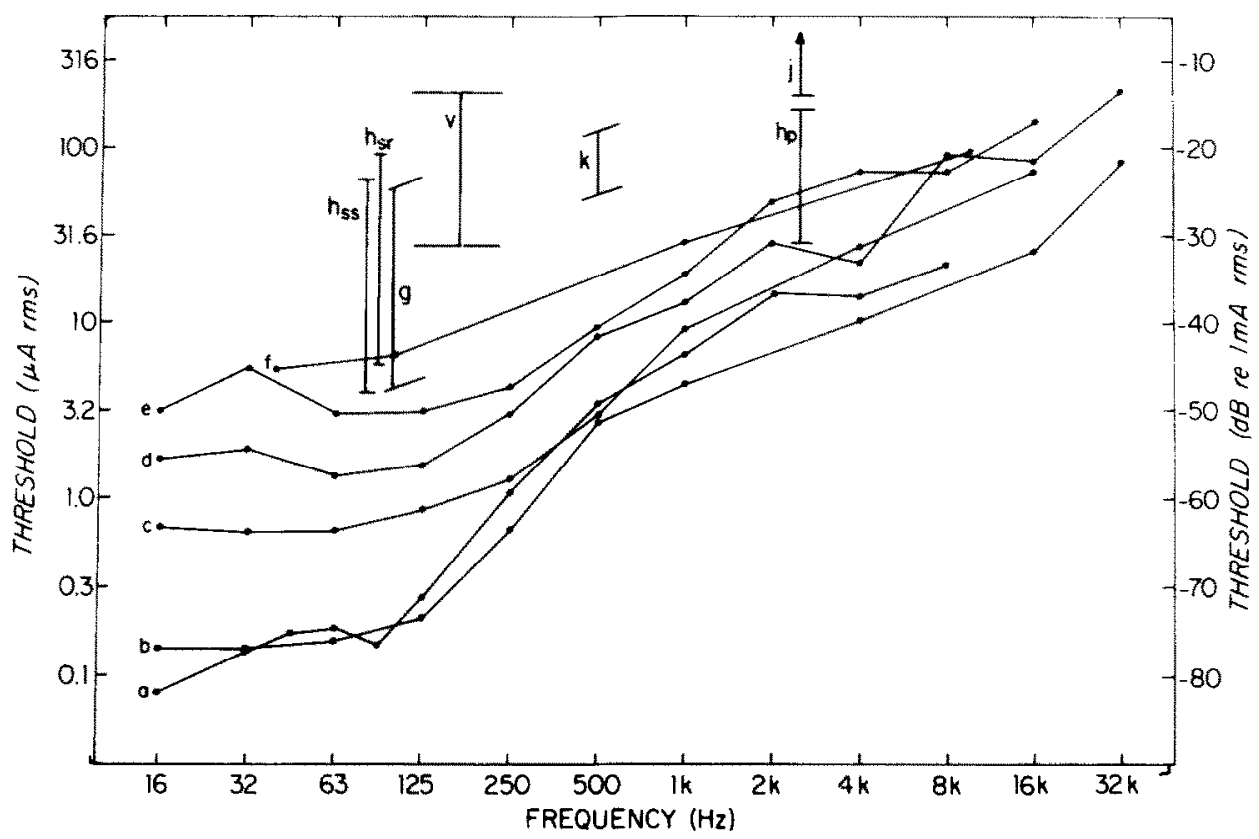

Fig. 7. Contours $a-f$ are psychophysical threshold contours for six monkeys from Fig. 1 . The range bars indicate the ranges of thresholds reported for auditory nerve fibers and cochlear nucleus neurons from cats and guinea pigs as described below. $g=$ Glass, 1983: AVCN, anesthetized guinea pigs, scala tympani electrodes with $1 \mathrm{~mm}$ separation, sinusoids, the cochlea was not treated with neomycin, 88 units, mode $=28 \mu$ Amp rms. $h=$ Hartmann et al., 1984: auditory nerve, anesthetized cats, round window monopolar stimulation. hss $=$ sinusoids, 'intact' cochleas, synchronization thresholds, mean $=26.3 \mu \mathrm{Amp}$ rms. hsr $=$ sinusoids, 'intact' cochleas, rate thresholds, mcan $=40.1 \mu \mathrm{Amp}$ rms. $\mathrm{hp}=0.2 \mathrm{msec} / \mathrm{phase}$ pulses, mechanically and chemically destroyed cochleas. $j=\mathrm{Javel}$ et al., 1987: auditory nerve, anesthetized cats, scala tympani electrodes with $0.75 \mathrm{~mm}$ separation, pulses, no neomycin. $k=\mathrm{Kiang}$ and Moxon, 1972: auditory nerve, anesthetized cat systemically treated with neomycin, round window to apex electrodes, sinusoids, 22 fibers. $b=$ van den Honert and Stypulkowski, 1984: auditory nerve, anesthetized cats, no neomycin, scala tympani tectrodes with 2 mm separation, pulses, 16 fibers, mean $=104 \mu$ Amp.

tion. This representation for pulses is based on observations that pulse width is the major determinant of psychophysical threshold with pulse rate playing a minor role under most circumstances (Eddington et al., 1978; Pfingst et al., 1979, 1980; Shannon, 1985). At pulse rates sufficient to produce a square wave, psychophysical thresholds for rectangular pulses are equivalent to those for sinusoids of equal period (Pfingst et al., 1980; Shannon, 1985). Represented in this way, the neural thresholds are consistently higher than the behavioral thresholds for comparable parameters.

A number of factors may be hypothesized to account for these differences, and given the large number of differences between the electrophysiological and behavioral experiments, the reasons for these differences cannot be resolved at this time. Possible contributors to the differences are single unit sampling bias, definitions of thresholds (Rose et al., 1971; Geisler et al., 1985), differences in electrode design and placement, differences in the waveforms used, and species differences. Physiological alteration of the preparation as required for single-unit studies as well as trauma due to the implant and/or procedures used to destroy hair cells must also be considered. In behavioral studies we have sometimes seen marked elevations in behavioral thresholds within the first few days after the implant surgery relative to the lower stable thresholds achieved several weeks after surgery (Pfingst et al., 1979), We hypothesize that these effects are due to temporary trauma from the implant and/or the deafening procedures. Clopton and Glass (1984) found that thresholds of ventral cochlear nucleus neurons were elevated in 
guinea pigs with neomycin treated cochleas as compared with those from animals with untreated cochleas.

The significance of these discrepancies between behavioral and neural thresholds depends on their cause. If they are due to species differences, differences in stimulation procedures, or differences in physiological treatment of the cochlea or the animal, these are matters which may simply help us better understand the important variables affecting electrical stimulation. On the other hand, if they are due to sampling bias or threshold definition, we must be concerned that the levels used in the physiological experiments may not be in a range appropriate for comparison with behavioral studies.

The basis of the discrepancies between behavioral and ncural thresholds might be partially resolved by making the psychophysical measurements and the neural measurements in the same animal at the same time using chronic single-unit recording techniques. This approach would eliminate all of the hypothesized causes of these differences except sampling bias and threshold definition.

Other interesting and potentially very useful comparisons can be made between behavioral and neural thresholds for electrical stimuli, though their interpretation depends to some extent on knowing the relationships of the actual levels used in each case. For example, the tuning curves for auditory nerve fibers (Kiang and Moxon, 1972) and anteroventral cochlear nucleus neurons (Glass, 1983) for sinusoidal electrical stimuli show some similarities to the behavioral threshold contours for these same stimuli. In both neural and psychophysical data, the maximum sensitivity (lowest threshold currents) is in the region of $100 \mathrm{~Hz}$. Above and below this region the thresholds for cochlear nucleus units increase at an average rate of about $4 \mathrm{~dB}$ /octave (Glass, 1983). Slopes of auditory nerve fibers increase at a similar or slightly lower rate (Kiang and Moxon, 1972). The slopes of the neural threshold contours above $100 \mathrm{~Hz}$, indicated by the sloping range bars for $g$ and $k$ in Fig. 7, are similar to the slopes of the behavioral threshold contours above $500 \mathrm{~Hz}$, but are much shallower than the slopes of most if not all of the behavioral threshold contours in the $100 \mathrm{~Hz}$ to $500 \mathrm{~Hz}$ re- gion. Furthermore, the behavioral threshold contours seldom rise as a function of decreasing frequency below $100 \mathrm{~Hz}$ as the neural tuning curves do. This suggests that some neural mechanism other than rate of discharge in single auditory neurons is involved in the detection of sinusoids in this frequency range, though once again questions of sampling bias, threshold definition, etc. must be considered.

\section{Frequency discrimination}

I will now turn to single-unit studies of frequency following in electrically stimulated auditory neurons and their relation to psychophysical studies of frequency discrimination. Investigators in several laboratories have found that neurons in the auditory periphery follow the frequency of sinusoidal electrical stimulation very well, perhaps better than they follow acoustic sinusoids (Hartmann et al., 1984; Glass, 1984). Hartmann has found that synchronization indices for electrical stimuli are on the average higher than those reported for acoustic stimuli by Johnson (1980) at almost all frequencies tested from $100 \mathrm{~Hz}$ to $3 \mathrm{kHz}$ (Hartmann, personal communication). This remarkably good frequency following behavior of auditory neurons contrasts markedly with the very poor frequency discrimination behavior of the organism (Figs. 5 and 6). This discrepancy suggests that the temporal information at the auditory periphery is not in a form which can by utilized by higher levels in the auditory pathway. Analyses such as Siebert's (1970) have indicated that the auditory nerve's frequency following even to acoustic stimulation is much better than would be predicted by frequency discrimination performance. This suggests that the auditory nerve may not be the most appropriate level to analyze the neural encoding of temporal information relative to perception. It is well known that temporal encoding (frequency following) deteriorates as the encoded information ascends the auditory pathway (Rouiller et al., 1979). At higher centers, the neural data might more closely match psychophysical performance (see Merzenich et al., 1973). Alternatively, this temporal information may become translated to a place code or other code at these centers, but the requirement of matching the neural limits to the psychophysical 
limits still applies. Given the reasonably close match between human and nonhuman primate performance on nonspectral frequency discrimination tasks, monkeys and possibly other animals as well, should serve as reasonable animal models to study the neural mechanisms of nonspectral pitch. Combined neural and psychophysical observations in the same subject would be an ideal model, providing the best index of the appropriate level of stimulation for the neural studies and providing the most unambiguous comparisons between the neural and behavioral measures.

\section{Conclusions}

The considerable overlap in results of psychophysical studies from implanted human and nonhuman primates suggests that the monkey is a good model for experimental studies applicable to humans. Whether or not this conclusion can be extended to other nonhuman mammals remains to be demonstrated. If neural data from animal studies are to be applied to humans, the discrepancies between the neural and psychophysical thresholds reported in the literature must be resolved. This can best be done by simultaneous psychophysical and neurophysiological studies in the same animal subjects. Such an experimental model would be ideal for assessing the validity of encoding models based on neural data. This powerful psychophysical-neural animal model applied to clinical problems should lead to significant advances in otolaryngology.

\section{Acknowledgements}

My thanks to Gregory Wakefield and an anonymous reviewer for helpful comments on earlier versions of this paper. Some of the work on nonhuman primates reviewed here was supported by NIH grant NS 21440.

\section{References}

Clopton, B.M. and Glass, I. (1984) Unit responses at cochlear nucleus to electrical stimulation through a cochlear prosthesis. Hear. Res. 14, 1-11.

Eddington, D.K., Dobelle, W.H., Brackmann, D.E., Mladejovsky, M.G. and Parkin, J.L. (1978) Auditory prosthesis research with multiple channel intracochlear stimulation in man. Ann. Otol. Rhinol. Laryngol. (St. Louis) 87, Supplement 53, 5-39.

Fay, R.R. (1988) Comparative psychoacoustics. Hear Res. 34, 295-306.

Formby, C. (1985) Differential sensitivity to tonal frequency and to the rate of amplitude modulation of broadband noise by normally hearing listeners. J. Acoust. Soc. Am. 78, 70-77.

Fourcin, A., Douek, E., Moore, B., Abberton, E., Rosen, S. and Waliker, J. (1984) Speech pattern element stimulation in electrical hearing. Arch. Otolaryngol. 110, 145-153.

Geisler, C.D., Deng, L. and Greenberg, S.R. (1985) Thresholds for primary auditory fibers using statistically defined criteria. J. Acoust. Soc. Am. 77, 1102-1109.

Glass, I. (1983) Tuning characteristics of cochlear nucleus units in response to electrical stimulation of the cochlea. Hear. Res. 12, 223-237.

Glass, I. (1984) Phase-locked responses of cochlear nucleus units to electrical stimulation through a cochlear implant. Exp. Brain Res. 55, 386-390.

Hartmann, R., Topp, G. and Klinke, R. (1984) Discharge patterns of cat primary auditory fibers with electrical stimulation of the cochlea. Hear. Res. 13, 47-62.

Hinojosa, R. and Marion, M. (1983) Histopathology of profound sensorineural deafness. Ann. N.Y. Acad. Sci. 405, $459-484$.

House, W.F. and Edgerton, B.J. (1982) A multiple-electrode cochlear implant. Ann. Otol. Rhinol. Laryngol. (St. Louis) 91, Supplement 91, 104-116.

Javel, E., Tong, Y.C., Shepherd, R.K. and Clark, G.M. (1987) Responses of cat auditory nerve fibers to biphasic electrical current pulses. Ann. Otol. Rhinol. Laryngol. (St. Louis) 96, Supplement 128, 26-30.

Johnson, D.H. (1980) The relationship between spike rate and synchrony in responses of auditory-nerve fibers to single tones. J. Acoust. Soc. Am. 68, 1115-1122.

Kiang, N.Y.S. and Moxon, E.C. (1972) Physiological considerations in artificial stimulation of the inner ear. Ann. Otol. Rhinol. Laryngol. (St. Louis) 81, 714-730.

Long, G.R. and Clark, W.W. (1984) Detection of frequency and rate modulation by the chinchilla. J. Acoust. Soc. Am. 75, 1184-1190.

Merzenich, M.M., Michelson, R.P., Pettit, C.R., Schindler, R.A. and Reid, M. (1973) Neural encoding of sound sensation evoked by electrical stimulation of the acoustic nerve. Ann. Otol. Rhinol. Laryngol. (St. Louis) 82, 486-503.

Moore, J.K. (1987) The human auditory brain-stem - a comparative view. Hear. Res. 29, 1-32.

Müller, C. (1981) Survey of cochlear implant work. J. Acoust. Soc. Am. 70, S52.

Nadol, J.B. Jr. (1988) Comparative anatomy of the cochlea and auditory nerve in mammals. Hear. Res. 34, 253-266.

Pfingst, B.E., Donaldson, J.A., Miller, J.M. and Spelman, F.A. (1979) Psychophysical evaluation of cochlear prosthesis in a monkey model. Ann. Otol. Rhinol. Laryngol. (St. Louis) 88, 613-625.

Pfingst, B.E., Spelman, F.A. and Sutton, D. (1980) Operating ranges for cochlear implants. Ann. Otol. Rhinol. Laryngol. (St. Louis) 89, Suppl. 66, 1-4. 
Pfingst, B.E., Burnett, P.A. and Sutton, D. (1983) Intensity discrimination with cochlear implants. J. Acoust. Soc. Am. 73, 1283-1292.

Pfingst, B.E. and Sutton, D. (1983) Relation of cochlear implant function to histopathology in monkeys. Ann. N.Y. Acad. Sci. 405, 224-239.

Pfingst, B.E. (1984) Operating ranges and intensity psychophysics for cochlear implants. Implications for speech processing strategies. Arch. Otolaryngol. 110, 140-144.

Pfingst, B.E., Evans, M. and Garrido, Y. (1984) Electrical frequency discrimination: Initial results from studies in macaques. Abstracts of the 1984 West Coast Cochlear Prosthesis Workshop, 18.

Pfingst, B.E., Glass, I., Spelman, F.A. and Sutton, D. (1985) Psychophysical studies of cochlear implants in monkeys: Clinical implications. In: R.A. Schindler, M.M. Merzenich (Eds.), Cochlear Implants Raven Press, New York, pp. 305-321.

Pfingst, B.E. and Rush, N.L. (1987) Discrimination of simultaneous frequency and level changes in electrical stimuli. Ann. Otol. Rhinol. Laryngol. (St. Louis) 96, Supplement 128, 34-37.

Rose, J.E., Hind, J.E., Anderson, D.J. and Brugge, J.F. (1971) Some effects of stimulus intensity on response of auditory nerve fibers in the squirrel monkey. J. Neurophysiol. 34, 685-699.

Rouiller, F., De Rihaupierre, Y. and De Ribaupierre, F. (1979)
Phase-locked responses to low frequency tones in the medial geniculate body. Hear Res. 1, 213-226.

Shannon, R.V. (1983) Electrical stimulation of the auditory nerve in man. I. Basic psychophysics. Hear. Res. 11, 157-189.

Shannon, R.V. (1985) Threshold and loudness function for pulsatile stimulation of cochlear implants. Hear. Res. 18, 135-143.

Siebert, W.M. (1970) Frequency discrimination in the auditory system: Place or periodicity mechanisms? Proc. IEEE 58, 723-730.

Sinnott, J.M., Petersen, M.R. and Hopp, S.L. (1985) Frequency and intensity discrimination in humans and monkeys. $\mathbf{J}$. Acoust. Soc. Am. 78, 1977-1985.

Spiegel, M.F. and Watson, C.S. (1984) Performance on frequency-discrimination tasks by musicians and nonmusicians. J. Acoust Soc. Am. 76, 1690-1695.

van den Honert, C. and Stypulkowski, P.H. (1984) Physiological properties of the electrically stimulated auditory nerve. II. Single fiber recordings. Hear. Res. 14, 225-243.

White, M.W. (1984) Psychophysical and neurophysiological considerations in the design of a cochlear prosthesis. Audiol. Ital. 1, 77-117.

Wier, C.C., Jesteadt, W. and Green, D.M. (1977) Frequency discrimination as a function of frequency and sensation level. J. Acoust Soc. Am. 61, 178-184. 